\title{
Closed-Loop Neural Network-Based NMES Control for Human Limb Tracking
}

\author{
Nitin Sharma, Member, IEEE, Chris M. Gregory, Marcus Johnson, Member, IEEE, and \\ Warren E. Dixon, Senior Member, IEEE
}

\begin{abstract}
Closed-loop control of skeletal muscle is complicated by the nonlinear muscle force to length and velocity relationships and the inherent unstructured and time-varying uncertainties in available models. Some pure feedback methods have been developed with some success, but the most promising and popular control methods for neuromuscular electrical stimulation (NMES) are neural network (NN)-based methods. Efforts in this paper focus on the use of a $\mathrm{NN}$ feedforward controller that is augmented with a continuous robust feedback term to yield an asymptotic result (in lieu of typical uniformly ultimately bounded stability). Specifically, an NN-based controller and Lyapunov-based stability analysis are provided to enable semi-global asymptotic tracking of a desired limb time-varying trajectory (i.e., non-isometric contractions). The developed controller is applied as an amplitude modulated voltage to external electrodes attached to the distal-medial and proximallateral portion of the quadriceps femoris muscle group in non-impaired volunteers. The added value of incorporating a NN feedforward term is illustrated through experiments that compare the developed controller with and without the NN feedforward component.
\end{abstract}

Index Terms-Asymptotic stability, closed-loop control of functional electrical stimulation (FES), neural networks (NNs), neuromuscular electrical stimulation (NMES), non-isometric contractions, nonlinear control, robust integral of the sign of the error (RISE)-based feedback.

\section{INTRODUCTION}

$\mathbf{N}$ EUROMUSCULAR ELECTRICAL STIMULATION (NMES) is a technique employed to generate desired muscle contractions via electrical stimulus [for functional tasks, NMES is described as functional electrical stimulation (FES)]. Efforts in NMES facilitate improved limb control and functionality for patients with stroke, spinal cord injuries, and other neurological impairments [1], [2]. Although most NMES procedures in physical therapy clinics consist of tabulated open-loop application of electrical stimulation, a market exists for the development of noninvasive closed-loop methods. NMES control development is hampered by several challenges

Manuscript received June 08, 2010; revised November 08, 2010; accepted February 08, 2011. Manuscript received in final form March 03, 2011. Date of publication April 05, 2011; date of current version April 11, 2012. Recommended by Associate Editor F. Chowdhury.

N. Sharma, M. Johnson, and W. E. Dixon are with the Department of Mechanical and Aerospace Engineering, University of Florida, Gainesville, FL 32611-6250 USA (e-mail: nitinsharma@ufl.edu; marc1518@ufl.edu; wdixon@ufl.edu.

C. M. Gregory is with the Brain Rehabilitation Research Center, North Florida/South Georgia Veterans Health System Department of Physical Therapy, University of Florida, Gainesville, FL 32611-6250 USA (e-mail: drewgreg@phhp.ufl.edu).

Digital Object Identifier 10.1109/TCST.2011.2125792 that affect the ability of a muscle to produce a desired force: muscle fatigue, hyperactive somatosensory reflexes, electrode placement, inter- and intra-subject variability in muscle properties, changing muscle geometry under the electrodes in non-isometric conditions, etc.

Some promising closed-loop experimental results have been reported that use high-gain linear feedback methods to compensate for uncertain muscle response (cf. [3]-[7] and the references therein). However, the development of analytical stability guarantees for linear feedback methods has been lacking due to the fact that the governing equations for muscle contractions are nonlinear with unstructured time-varying uncertainties. Feedback techniques such as linear quadratic Gaussian (LQG) methods, gain scheduling methods, and pole placement methods were developed and analyzed under a linear muscle model assumption [8]-[10]. Recently, nonlinear robust techniques such as sliding mode control (SMC) (cf. [11], [12]) and robust integral of the sign of the error (RISE) [13] methods have been developed and analyzed for uncertain nonlinear muscle models. Although stability results can be achieved for representative nonlinear muscle models, these results, as well as previous linear feedback methods, inherently rely on high gains or high frequency to dominate the model uncertainty, potentially resulting in overstimulation.

Seminal work in [14]-[19] continue to inspire new investigations (cf. [20]-[25] and the references within) in neural network (NN)-based NMES control development. One motivation for NN-based controllers is the desire to augment feedback methods with an adaptive element that can adjust to the uncertain muscle model, rather than only relying on feedback to dominate the uncertainty based on worse case scenarios. NN-based control methods have attracted more attention in NMES than other adaptive feedforward methods because of the nature of the unstructured uncertainty and the universal approximation property of NNs. However, since NNs can only approximate a function within some residual approximation error, all previous NN-based controllers yield uniformly ultimately bounded stability (i.e., the errors converge to a region of bounded steady-state error).

Our previous result in [13] focuses on the development of a RISE-based NMES controller and the associated analytical stability analysis that yields asymptotic tracking in the presence of a nonlinear uncertain muscle model with nonvanishing additive disturbances. The result in [13] uses feedback and an implicit learning mechanism to dominate uncertainty and disturbances. Recent results from general control systems literature [26] indicate that the RISE-based feedback structure can be augmented 
with a NN feedforward term to yield asymptotic tracking for some classes of systems. Based on these general results (and our preliminary work in [27]), the RISE-based method in [13] is modified with an NN to develop a new NMES controller for the uncertain muscle model. The developed controller is applied as an amplitude modulated voltage to external electrodes attached to the distal-medial and proximal-lateral portion of the quadriceps femoris muscle group in non-impaired volunteers. The experimental results indicate that the addition of the $\mathrm{NN}$ to the RISE controller reduces the root mean squared (RMS) tracking error for similar RMS voltage when compared to the method in [13] without the NN feedforward component (RISE controller alone).

\section{Muscle Activation AND Limb Model}

The musculoskeletal dynamics with one-degree of rotational freedom about the knee joint is given as [6]

$$
M_{I}+M_{e}+M_{g}+M_{v}+\tau_{d}=\tau
$$

In (1), $M_{I}(\ddot{q}) \in \mathbb{R}$ denotes the inertia of the shank-foot complex about the knee-joint, $M_{e}(q) \in \mathbb{R}$ denotes elastic effects due to joint stiffness, $M_{g}(q) \in \mathbb{R}$ denotes the gravitational component, $M_{v}(\dot{q}) \in \mathbb{R}$ denotes viscous effects due to damping in the musculotendon complex [28], $\tau_{d}(t) \in \mathbb{R}$ represents unknown unmodeled bounded disturbances (e.g., fatigue, signal, and response delays, spasms, changing muscle geometry), and $\tau(t) \in \mathbb{R}$ denotes the torque produced at the knee joint by the electric potential.

The inertia and gravitational effects in (1) can be modelled as

$$
M_{I}=J \ddot{q}, \quad M_{g}=m g l \sin (q)
$$

where $q(t), \dot{q}(t), \ddot{q}(t) \in \mathbb{R}$ denote the angular position, velocity, and acceleration of the lower shank about the knee-joint, respectively, $J \in \mathbb{R}$ denotes the unknown inertia of the combined shank and foot, $m \in \mathbb{R}$ denotes the unknown combined mass of the shank and foot, $l \in \mathbb{R}$ is the unknown distance between the knee-joint and the lumped center of mass of the shank and foot, and $g \in \mathbb{R}$ denotes the gravitational acceleration. The elastic effects are modelled on the empirical findings by Ferrarin and Pedotti in [28] as

$$
M_{e}=k_{1}\left(\exp \left(-k_{2} q\right)\right)\left(q-k_{3}\right)
$$

where $k_{1}, k_{2}, k_{3} \in \mathbb{R}$ are unknown positive coefficients. As shown in [6], the viscous moment $M_{v}(\dot{q})$ can be modelled as

$$
M_{v}=B_{1} \tanh \left(-B_{2} \dot{q}\right)-B_{3} \dot{q}
$$

where $B_{1}, B_{2}$, and $B_{3} \in \mathbb{R}$ are unknown positive constants.

The torque produced about the knee is controlled through muscle forces that are elicited by NMES. For simplicity and without loss of generality, the subsequent development focuses on producing knee torque through muscle tendon forces generated by electrical stimulation of the quadriceps (i.e., antagonistic muscle forces are not considered). The knee torque is related to the muscle tendon force $F(q, \dot{q}, t) \in \mathbb{R}$ as

$$
\tau=\zeta F
$$

where $\zeta(q) \in \mathbb{R}$ denotes a positive moment arm that changes with the extension and flexion of the leg as shown in studies by [29] and [30]. The total muscle force is a sum of active force generated by contractile element (often denoted as $F_{\mathrm{CE}}$ ), the tension generated by passive elastic elements (often denoted as $F_{\mathrm{PE}}$ ) and the forces generated by viscous fluids (often denoted as $\left.F_{\mathrm{VE}}\right)$. The muscle force generated at the tendon is the projection of net sum of these elements along the line parallel to the tendon. The total muscle force generated at the tendon is considered a function of the unknown nonlinear function $\eta(q, \dot{q}) \in \mathbb{R}$ and voltage $V(t)$ applied to the quadriceps muscle by electrical stimulation defined as

$$
F=\eta V \text {. }
$$

The introduction of the unknown nonlinear function $\eta(q, \dot{q})$ enables the muscle contraction to be considered under general dynamic conditions in the subsequent control development. The uncertain and unknown function $\eta(q, \dot{q})$ captures the dynamic characteristics of muscle recruitment (approximated by a continuously differentiable function), and active and passive muscle characteristics. The active and passive characteristics include increase in elastic element with increasing muscle length and muscle stiffness changes of potentially more than two orders of magnitude [31] under dynamic contractions.

The model developed in (1)-(5) is used to examine the stability of the subsequently developed controller, but the controller does not explicitly depend on these models. Specifically, an $\mathrm{NN}$ is used to approximate the muscle dynamics along with the implicit learning of the RISE feedback structure. The following assumptions are used to facilitate the subsequent control development and stability analysis.

Assumption 1: The moment arm $\zeta(q)$ is assumed to be a non-zero, positive, bounded function [29], [30] whose first two time derivatives exist. Based on the empirical data in [32] and [33], the function $\eta(q, \dot{q})$ is assumed to be a non-zero, positive, and bounded function with bounded first and second time derivatives.

Assumption 2: The auxiliary non-zero unknown scalar function $\Omega(q, \dot{q}) \in \mathbb{R}$ is defined as

$$
\Omega=\zeta \eta
$$

where the first and second time derivatives of $\Omega(q, \dot{q})$ are assumed to exist and be bounded (see Assumption 1).

Assumption 3: The unknown disturbance $\tau_{d}(t)$ is bounded and its first and second derivatives with respect to time exist and are bounded. Based on Assumptions 1 and 2, the ratio $\tau_{d}(t) / \Omega(q, \dot{q})$ is also assumed to be bounded and its first and second derivatives with respect to time exist and are bounded.

\section{Control DeVelopment and Stability ANAlysis}

The objective is to develop an NMES controller to produce a desired torque at the knee to enable the knee angle to track a desired trajectory, denoted by $q_{d}(t) \in \mathbb{R}$. The desired trajectory can be any continuous signal (or a simple constant setpoint). In the subsequent experimental results the desired signal is a sinusoidal trajectory. The sinusoidal trajectory is arbitrary and may 
not correspond to functional trajectory, but the period of the sinusoid is motivated by the speed of typical walking gaits. Although such trajectories may not correspond to functional trajectories, the ability to track arbitrary trajectories is necessary for the performance of many functional tasks elicited through external electrical stimulation. To quantify the objective, a limb position tracking error, denoted by $e_{1}(t) \in \mathbb{R}$, is defined as

$$
e_{1}=q_{d}-q
$$

where $q_{d}(t)$ is an a priori trajectory which is designed such that $q_{d}(t)$ and $q_{d}^{i}(t)$ are bounded and within the knee range of motion, where $q_{d}^{i}(t)$ denotes the $i$ th derivative for $i=1,2,3,4$. To facilitate the subsequent analysis, filtered tracking errors, denoted by $e_{2}(t)$ and $r(t) \in \mathbb{R}$, are defined as

$$
e_{2}=\dot{e}_{1}+\alpha_{1} e_{1}, \quad r=\dot{e}_{2}+\alpha_{2} e_{2}
$$

where $\alpha_{1}, \alpha_{2} \in \mathbb{R}$ denote positive constants. The filtered tracking error $r(t)$ is introduced to facilitate the closed-loop error system development and stability analysis but is not used in the controller because of a dependence on acceleration measurements.

\section{A. Open-Loop Error System Development}

The open-loop tracking error system can be developed by multiplying (8) by $J / \Omega(q, \dot{q})$ and by utilizing the expressions in (1) and (4)-(8) as

$$
J_{\Omega} r=J_{\Omega}\left(\alpha_{2} e_{2}+\alpha_{1} \dot{e}_{1}+\ddot{q}_{d}\right)+L_{\Omega}-V+\tau_{d \Omega}
$$

where $J_{\Omega}(q, \dot{q}) \in \mathbb{R}, L_{\Omega}(q, \dot{q}) \in \mathbb{R}$, and $\tau_{d \Omega}(q, \dot{q}) \in \mathbb{R}$ are defined as

$$
J_{\Omega}=\frac{J}{\Omega}, \quad L_{\Omega}=\frac{1}{\Omega}\left(M_{e}+M_{g}+M_{v}\right), \quad \tau_{d \Omega}=\frac{\tau_{d}}{\Omega} .
$$

To facilitate the subsequent analysis, auxiliary signals $J_{\Omega d}\left(q_{d}, \dot{q}_{d}\right) \in \mathbb{R}$ and $L_{\Omega d}\left(q_{d}, \dot{q}_{d}\right) \in \mathbb{R}$ are defined as in (10) where the functional dependencies on $q(t)$ and $\dot{q}(t)$ are replaced with $q_{d}(t)$ and $\dot{q}_{d}(t)$. By adding and subtracting $f_{d}\left(q_{d}, \dot{q}_{d}, \ddot{q}_{d}\right) \in \mathbb{R}$, defined as

$$
f_{d}=L_{\Omega d}+J_{\Omega d} \ddot{q}_{d}
$$

the dynamics in (9) can be rewritten as

$$
J_{\Omega} r=f_{d}+S-V+\tau_{d \Omega}
$$

where the auxiliary function $S\left(q, q_{d}, \dot{q}, \dot{q}_{d}, \ddot{q}_{d}\right) \in \mathbb{R}$ is defined as

$$
S=J_{\Omega}\left(\alpha_{2} e_{2}+\alpha_{1} \dot{e}_{1}\right)+J_{\Omega} \ddot{q}_{d}-J_{\Omega d} \ddot{q}_{d}+L_{\Omega}-L_{\Omega d} .
$$

\section{B. Feedforward NN Estimation}

NN-based estimation methods are well suited for NMES because the muscle model contains unstructured nonlinear disturbances as given in (1) (i.e., uncertainties that do not satisfy the linear-in-the-parameters assumption). Let $\mathbb{S}$ be a compact simply connected set of $\mathbb{R}^{4}$. Let $\mathbb{C}(\mathbb{S})$ be defined as the space where $f_{d}\left(x_{d}\right): \mathbb{S} \rightarrow \mathbb{R}$ is continuous. The universal approximation property states that there exist weights and thresholds such that the function $f_{d}\left(x_{d}\right) \in \mathbb{C}(\mathbb{S})$ can be represented by a three-layer NN as [34]

$$
f_{d}=W^{T} \sigma\left(U^{T} x_{d}\right)+\epsilon\left(x_{d}\right)
$$

where $x_{d}(t) \in \mathbb{R}^{4}$ is defined as $x_{d}(t)=\left[1 q_{d}(t) \dot{q}_{d}(t) \ddot{q}_{d}(t)\right]^{T}$. In (13), $U \in \mathbb{R}^{4 \times N_{1}}$ and $W \in \mathbb{R}^{N_{1}+1}$ are bounded constant ideal weight matrices for the first-to-second and second-to-third layers, respectively, where $N_{1}$ is the number of neurons in the hidden layer. The sigmoid activation function in (13) is denoted by $\sigma(\cdot): \mathbb{R}^{4} \rightarrow \mathbb{R}^{N_{1}+1}$, and $\epsilon\left(x_{d}\right): \mathbb{R}^{4} \rightarrow \mathbb{R}$ is the functional reconstruction error. The additional term " 1 " in the input vector $x_{d}(t)$ and activation term $\sigma(\cdot)$ allows for thresholds to be included as the first columns of the weight matrices [34]. Thus, any estimation of $W$ and $U$ then includes estimation of the thresholds. Based on (13), the typical three layer NN approximation for $f_{d}\left(x_{d}\right)$ is given as [34]

$$
\hat{f}_{d}=\hat{W}^{T} \sigma\left(\hat{U}^{T} x_{d}\right)
$$

where $\hat{U}(t) \in \mathbb{R}^{4 \times N_{1}}$ and $\hat{W}(t) \in \mathbb{R}^{N_{1}+1}$ are subsequently designed estimates of the ideal weight matrices. The estimate mismatch for the ideal weight matrices, denoted by $\tilde{U}(t) \in \mathbb{R}^{4 \times N_{1}}$ and $\tilde{W}(t) \in \mathbb{R}^{N_{1}+1}$, are defined as

$$
\tilde{U}=U-\hat{U}, \quad \tilde{W}=W-\hat{W}
$$

and the mismatch for the hidden-layer output error, denoted by $\tilde{\sigma}\left(x_{d}\right) \in \mathbb{R}^{N_{1}+1}$, is defined as

$$
\tilde{\sigma}=\sigma-\hat{\sigma}=\sigma\left(U^{T} x_{d}\right)-\sigma\left(\hat{U}^{T} x_{d}\right)
$$

Assumption 4 (Boundedness of the Ideal Weights): The ideal weights are assumed to exist and are bounded by known positive values so that

$$
\begin{gathered}
\|U\|_{F}^{2}=\operatorname{tr}\left(U^{T} U\right)=\operatorname{vec}(U)^{T} \operatorname{vec}(U) \leq \bar{U}_{B} \\
\|W\|_{F}^{2}=\operatorname{tr}\left(W^{T} W\right)=\operatorname{vec}(W)^{T} \operatorname{vec}(W) \leq \bar{W}_{B}
\end{gathered}
$$

where $\|\cdot\|_{F}$ is the Frobenius norm of a matrix, and $\operatorname{tr}(\cdot)$ is the trace of a matrix. The ideal weights in an NN are bounded, but knowledge of this bound is a non-standard assumption in typical NN literature (although this assumption is also used in textbooks such as [34]). If the ideal weights are constrained to stay within some predefined threshold, then the function reconstruction error will be larger. Typically, this would yield a larger ultimate steady-state bound. Yet, in the current result, the mismatch resulting from limiting the magnitude of the weights is compensated through the RISE feedback structure (i.e., the RISE structure eliminates the disturbance due to the function reconstruction error). Based on the assumption that the desired trajectory is bounded, the following inequalities hold:

$$
\left|\epsilon\left(x_{d}\right)\right| \leq \epsilon_{b_{1}} \quad\left|\dot{\epsilon}\left(x_{d}\right)\right| \leq \epsilon_{b_{2}} \quad\left|\ddot{\epsilon}\left(x_{d}\right)\right| \leq \epsilon_{b_{3}}
$$

where $\epsilon_{b_{1}}, \epsilon_{b_{2}}$ and $\epsilon_{b_{3}} \in \mathbb{R}$ are known positive constants. 
Remark 1: One motivation to add and subtract the auxiliary function $f_{d}\left(q_{d}, \dot{q}_{d}, \ddot{q}_{d}\right)$ to yield (12) is to develop the input vector $x_{d}(t)$ in terms of the desired trajectory, thus avoiding higher order state derivatives in the $\mathrm{NN}$ input vector and ensuring that $f_{d}\left(q_{d}, \dot{q}_{d}, \ddot{q}_{d}\right)$ is defined on $\mathbb{S}$.

\section{Closed-Loop Error System Development}

The control development in this section is motivated by several technical challenges associated with blending the NN feedforward term with the RISE feedback method. One of the challenges to use the RISE control structure is that an extra time derivative of the dynamics, which generates acceleration dependent terms, is used in the stability analysis. If the $\mathrm{NN}$ is a function of the actual system states, the NN update laws will require acceleration measurements. To avoid the use of acceleration measurements, the NN structure in (14) is developed in terms of the desired trajectories. Another challenge is that, while the NN estimate are upper bounded by constant, the time derivatives of these terms are state dependent, and hence violate the traditional RISE assumptions. To address this issue, the closed-loop error system development requires a strategic separation and regrouping of terms. In this section, the control is designed and the closed-loop error system is presented.

Based on the open-loop error system in (12) and the subsequent stability analysis (see the development in Appendix A), the control torque input is designed as [26]

$$
V=\hat{f}_{d}+\mu
$$

where $\hat{f}_{d}(t) \in \mathbb{R}$ is the three-layer NN feedforward estimate defined in (14), and $\mu(t) \in \mathbb{R}$ is the RISE feedback term designed as [35]-[37]

$$
\mu \triangleq\left(k_{s}+1\right) e_{2}(t)-\left(k_{s}+1\right) e_{2}(0)+\nu .
$$

In (21), $k_{s} \in \mathbb{R}$ denotes positive constant adjustable control gain, and $\nu(t) \in \mathbb{R}$ is the generalized solution to

$$
\dot{\nu}=\left(k_{s}+1\right) \alpha_{2} e_{2}(t)+\beta_{1} \operatorname{sgn}\left(e_{2}(t)\right), \quad \nu(0)=0
$$

where $\beta_{1} \in \mathbb{R}$ denotes positive constant adjustable control gain, and $\operatorname{sgn}(\cdot)$ denotes the signum function. The estimates for the $\mathrm{NN}$ weights in (14) are generated online using a projection algorithm as

$$
\begin{aligned}
\dot{\hat{W}} & =\operatorname{proj}\left(\Gamma_{1} \hat{\sigma}^{\prime} \hat{U}^{T} \dot{x}_{d} e_{2}^{T}\right) \\
\dot{\hat{U}} & =\operatorname{proj}\left(\Gamma_{2} \dot{x}_{d}\left(\hat{\sigma}^{\prime T} \hat{W} e_{2}\right)^{T}\right)
\end{aligned}
$$

where $\Gamma_{1} \in \mathbb{R}^{\left(N_{1}+1\right) \times\left(N_{1}+1\right)}$ and $\Gamma_{2} \in \mathbb{R}^{4 \times 4}$ are constant, positive definite, symmetric gain matrices. The NN-based feedforward component $\hat{f}_{d}(t)$ is used to approximate the desired musculoskeletal dynamics $f_{d}\left(q_{d}, \dot{q}_{d}, \ddot{q}_{d}\right)$ given in (11). The NN component approximates the desired function through adaptive weight estimates that are adjusted online via the adaptive law given in (23). The RISE feedback controller $\mu(t)$ has implicit learning characteristics [37] which maintains the robustness of the system in the presence of additive disturbances and residual function approximation error. Also during the transient response of the trial, the role of the RISE feedback controller is to keep the system stable while the NN approximates the system dynamics.

The closed-loop tracking error system can be developed by substituting (20) into (12) as

$$
J_{\Omega} r=\tilde{f}_{d}+S-\mu+\tau_{d \Omega}
$$

where

$$
\tilde{f}_{d}=f_{d}-\hat{f}_{d}
$$

To facilitate subsequent closed-loop stability analysis, the time derivative of (24) can be determined as

$$
J_{\Omega} \dot{r}=-\dot{J}_{\Omega} r+\dot{\tilde{f}}_{d}+\dot{S}-\dot{\mu}+\dot{\tau}_{d \Omega} .
$$

Although the voltage control input $V(t)$ is present in the openloop error system in (12), an additional derivative is taken to facilitate the design of the RISE-based feedback controller. After substituting the time derivative of (25) into (26) by using (13) and (14), the closed-loop system can be expressed as

$$
\begin{aligned}
J_{\Omega} \dot{r}= & -\dot{J}_{\Omega} r+W^{T} \sigma^{\prime}\left(U^{T} x_{d}\right) U^{T} \dot{x}_{d}-\dot{\hat{W}}^{T} \sigma\left(\hat{U}^{T} x_{d}\right) \\
& -\hat{W}^{T} \sigma^{\prime}\left(\hat{U}^{T} x_{d}\right) \hat{U}^{T} \dot{x}_{d}-\hat{W}^{T} \sigma^{\prime}\left(\hat{U}^{T} x_{d}\right) \dot{\hat{U}}^{T} x_{d} \\
& +\dot{\epsilon}\left(x_{d}\right)+\dot{S}-\dot{\mu}+\dot{\tau}_{d \Omega}
\end{aligned}
$$

where $\sigma^{\prime}\left(\hat{U}^{T} x_{d}\right)=d \sigma\left(U^{T} x_{d}\right) /\left.d\left(U^{T} x_{d}\right)\right|_{U^{T} x_{d}=\hat{U}^{T} x_{d}}$. After adding and subtracting the terms $W^{T} \hat{\sigma}^{\prime} \hat{U}^{T} \dot{x}_{d}+\hat{W}^{T} \hat{\sigma} \tilde{U}^{T} \dot{x}_{d}$ to (27), the following expression can be obtained:

$$
\begin{aligned}
J_{\Omega} \dot{r}= & -\dot{J}_{\Omega} r+\hat{W}^{T} \hat{\sigma}^{\prime} \tilde{U}^{T} \dot{x}_{d}+\tilde{W}^{T} \hat{\sigma}^{\prime} \hat{U}^{T} \dot{x}_{d} \\
& -\hat{W}^{T} \hat{\sigma}^{\prime} \tilde{U}^{T} \dot{x}_{d}-W^{T} \hat{\sigma}^{\prime} \hat{U}^{T} \dot{x}_{d} \\
& +W^{T} \sigma^{\prime} U^{T} \dot{x}_{d}+\dot{\epsilon}\left(x_{d}\right)-\hat{W}^{T} \hat{\sigma}^{\prime} \dot{\hat{U}}^{T} x_{d} \\
& -\dot{\hat{W}}^{T} \hat{\sigma}+\dot{S}-\dot{\mu}+\dot{\tau}_{d \Omega}
\end{aligned}
$$

where the notation $\hat{\sigma}(\cdot)$ is introduced in (16). Using the NN weight tuning laws described in (23), the expression in (28) can be rewritten as

$$
J_{\Omega} \dot{r}=-\frac{1}{2} \dot{J}_{\Omega} r+\tilde{N}+N-e_{2}-\left(k_{s}+1\right) r-\beta \operatorname{sgn}\left(e_{2}\right)
$$

where the unmeasurable auxiliary terms $\tilde{N}\left(e_{1}, e_{2}, r, t\right)$ and $N\left(\hat{W}, \hat{U}, x_{d}, \dot{x}_{d}, t\right) \in \mathbb{R}$ given in (29) are defined as

$$
\begin{aligned}
\tilde{N}= & -\frac{1}{2} \dot{J}_{\Omega} r+\dot{S}+e_{2}-\operatorname{proj}\left(\Gamma_{1} \hat{\sigma}^{\prime} \hat{U}^{T} \dot{x}_{d} e_{2}^{T}\right)^{T} \hat{\sigma} \\
& -\hat{W}^{T} \hat{\sigma}^{\prime} \operatorname{proj}\left(\Gamma_{2} \dot{x}_{d}\left(\hat{\sigma}^{\prime T} \hat{W} e_{2}\right)^{T}\right)^{T} x_{d} \\
N= & N_{B}+N_{d} .
\end{aligned}
$$

In (31), $N_{d}\left(x_{d}, \dot{x}_{d}, t\right) \in \mathbb{R}$ is defined as

$$
N_{d}=W^{T} \sigma^{\prime} U^{T} \dot{x}_{d}+\dot{\epsilon}\left(x_{d}\right)+\dot{\tau}_{d \Omega}
$$

while $N_{B}\left(\hat{W}, \hat{U}, x_{d}, \dot{x}_{d}, t\right) \in \mathbb{R}$ is defined as

$$
N_{B}=N_{B_{1}}+N_{B_{2}}
$$


where $N_{B_{1}}\left(\hat{W}, \hat{U}, x_{d}, \dot{x}_{d}, t\right)$ and $N_{B_{2}}\left(\hat{W}, \hat{U}, x_{d}, \dot{x}_{d}, t\right) \in \mathbb{R}$ are defined as

$$
N_{B_{1}}=-\hat{W}^{T} \hat{\sigma}^{\prime} \tilde{U}^{T} \dot{x}_{d}-W^{T} \hat{\sigma}^{\prime} \hat{U}^{T} \dot{x}_{d}
$$

and

$$
N_{B_{2}}=\hat{W}^{T} \hat{\sigma}^{\prime} \tilde{U}^{T} \dot{x}_{d}+\tilde{W}^{T} \hat{\sigma}^{\prime} \hat{U}^{T} \dot{x}_{d}
$$

Motivation for the definitions in (30)-(32) are based on the need to segregate terms that are bounded by state-dependent bounds and terms that are upper bounded by constants for the development of the NN weight update laws and the subsequent stability analysis. The auxiliary term in (33) is further segregated to develop gain conditions in the stability analysis. Based on the segregation of terms in (30), the mean value theorem can be applied to upper bound $\tilde{N}\left(e_{1}, e_{2}, r, t\right)$ as

$$
|\tilde{N}| \leq \rho(\|z\|)\|z\|
$$

where $z(t) \in \mathbb{R}^{3}$ is defined as

$$
z \triangleq\left[e_{1} e_{2} r\right]^{T}
$$

and the bounding function $\rho(\|z\|) \in \mathbb{R}$ is a positive globally invertible nondecreasing function. Based on Assumption 3, (17)-(19), and (33)-(35), the following inequalities can be developed [26]:

$$
\left|N_{d}\right| \leq \zeta_{1} \quad\left|N_{B}\right| \leq \zeta_{2} \quad\left|\dot{N}_{d}\right| \leq \zeta_{3} \quad\left|\dot{N}_{B}\right| \leq \zeta_{4}+\zeta_{5}\left|e_{2}\right|
$$

where $\zeta_{i} \in \mathbb{R},(i=1,2, \ldots 5)$ are known positive constants.

Theorem 1: The composite NN and RISE controller given in (20)-(22) ensures that all system signals are bounded under closed-loop operation and that the position tracking error is regulated in the sense that

$$
\left|e_{1}(t)\right| \rightarrow 0 \quad \text { as } t \rightarrow \infty
$$

provided the control gains in (8), (21), and (22) are selected according to the following sufficient conditions:

$$
\begin{aligned}
\alpha_{1} & >\frac{1}{2}, \quad \alpha_{2}>\beta_{2}+1 \\
\beta_{1} & >\zeta_{1}+\zeta_{2}+\frac{1}{\alpha_{2}} \zeta_{3}+\frac{1}{\alpha_{2}} \zeta_{4}, \quad \beta_{2}>\zeta_{5}
\end{aligned}
$$

and control gain $k_{s}$ defined in (21) is chosen sufficiently large based on the initial conditions of the error system, where $\zeta_{i} \in$ $\mathbb{R},(i=1,2, \ldots, 5)$ are known positive constants defined in (38), and $\beta_{2} \in \mathbb{R}$ is a subsequently defined positive constant.

Proof: See Appendix A.

\section{EXPERIMENTAL RESULTS}

Experimental results obtained with volunteer subjects are provided in this section to examine the performance of the developed controller given in (20)-(22). These results were compared with the previous results in [13] that used the RISE feedback structure without the NN feedforward term. The
NMES controller was implemented as an amplitude modulated voltage composed of a positive rectangular pulse with a fixed width of $400 \mu \mathrm{sec}$ and fixed frequency of $30 \mathrm{~Hz}$. The a priori chosen stimulation parameters are within the ranges typically reported during NMES studies [13]. Without loss of generality, the developed controller is applicable to different stimulation protocols (i.e., voltage, frequency, or pulse width modulation). The following results indicate that the developed controller (henceforth denoted as NN+RISE) was able to minimize the knee angle error while dynamically tracking a desired trajectory.

\section{A. Testbed and Protocol}

Three sets of experiments were conducted including tracking experiments, regulation experiments, and a sit-to-stand tracking experiment. The objective in tracking experiments (including the sit-to-stand tracking case) was to enable the knee and lower leg to follow an angular trajectory, whereas, the objective of regulation experiments was to regulate the knee and lower leg to a constant desired setpoint. For tracking and regulation experiments, the testbed consists of a custom computer controlled stimulation circuit and a modified leg extension machine (LEM). The LEM was modified to include optical encoders. The LEM allows seating adjustments to ensure the rotation of the knee is about the encoder axis. A $4.5 \mathrm{~kg}(10 \mathrm{lb})$ load was attached to the weight bar of the LEM and a mechanical stop was used to prevent hyperextension. The sit-to-stand tracking experiment was performed to illustrate the controller performance in a more functional weight bearing task where the person was not sitting in the LEM. For this experiment, a person was seated in a chair while leaning forward (so the center of gravity would be positioned to enable the person to stand via leg extension). The person's knee angle was measured using a goniometer (manufactured by Biometrics Ltd.) attached to both sides of the knee joint. The goniometer was interfaced with the custom computer controlled stimulation circuit via an angle display unit (ADU301). For all experiments, bipolar self-adhesive neuromuscular stimulation electrodes were placed over the distal-medial and proximal-lateral portion of the quadriceps femoris muscle group of volunteers and connected to custom stimulation circuitry. For each experiment, the computed voltage input was modulated by a fixed pulse width of $400 \mu \mathrm{s}$ and fixed frequency of $30 \mathrm{~Hz}$. The stimulation frequency was selected based on subject comfort and to minimize fatigue.

The experiments were conducted on nine non-impaired subjects including eight males and one female (as in our previous study in [13]) with age ranges of 20 to 35 years, with written informed consent as approved by the Institutional Review Board at the University of Florida. The electrical stimulation responses of non-impaired subjects have been reported as similar to paraplegic subjects' responses [11], [17], [21]. Volunteers were instructed to relax as much as possible and to allow the stimulation to control the limb motion (i.e., the subject was not supposed to influence the leg motion voluntarily and was not allowed to see the desired trajectory). In the first set of experiments, the 

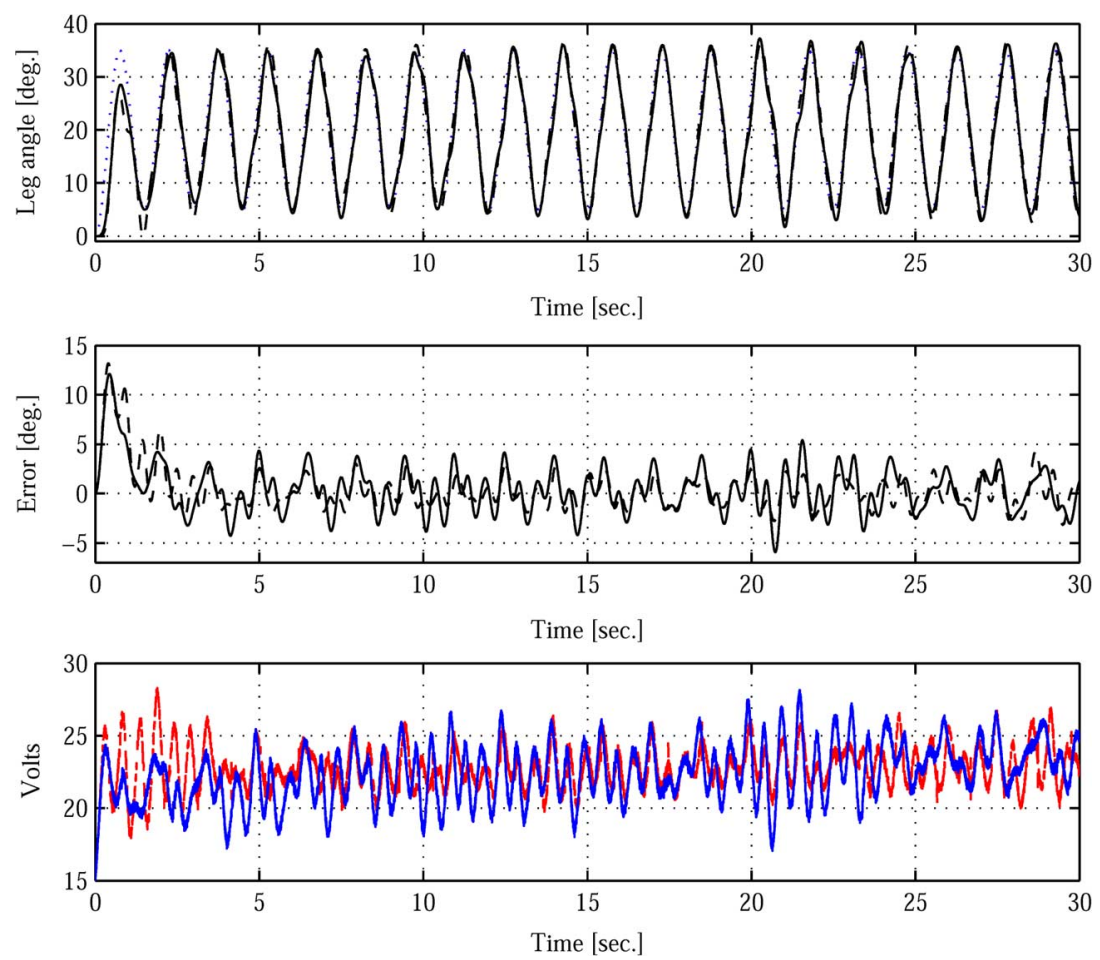

Fig. 1. Top plot shows the actual limb trajectories obtained from the NN+RISE controller (dashed line) and the RISE controller (solid line) versus the desired 1.5 period trajectory (dotted line). The middle plot shows the tracking error (desired angle minus actual angle) obtained from NN+RISE (dashed line) and RISE (solid line) controllers. The maximum steady state errors obtained are $4.24^{\circ}$ (at $28.6 \mathrm{~s}$ ) and $5.95^{\circ}$ (at $20.7 \mathrm{~s}$ ) for NN+RISE and RISE controller, respectively. The bottom plot shows the computed NN+RISE voltage (dashed line) and RISE voltage (solid line). The maximum steady state voltage obtained are 26.95 (at $29.1 \mathrm{~s}$ ) and $28.1 \mathrm{~V}$ (at $21.47 \mathrm{~s}$ ) for NN+RISE and RISE controller, respectively.

study was conducted for different types of desired trajectories including: a $1.5 \mathrm{~s}$ periodic trajectory and a dual periodic trajectory (combined 4 and $6 \mathrm{~s}$ periods). Controllers were implemented on both legs of four subjects using the trajectory with a $1.5 \mathrm{~s}$ period, while the rest of the tests were performed on only one leg of the other three subjects since they were not available for further testing. Three subjects [one male, one female (both legs); one male (one leg)] were asked to volunteer for the dual periodic desired trajectory tests. The regulation tests were performed on one of the legs of two subjects, while the sit-to-stand experiment was performed on one healthy normal individual. Each subject participated in one trial per criteria (e.g., one result was obtained in a session for a given desired trajectory). A pretrial test was performed on each volunteer in each experimental session to find the appropriate initial voltage for the controller to reduce the initial transient error. After the pretrial test, the RISE controller was implemented on each subject for a thirty second duration and its performance was recorded. A rest period of five minutes was provided before the NN+RISE controller was implemented for an additional thirty second duration. The $\mathrm{NN}+\mathrm{RISE}$ controller was implemented with three input layer neurons, 25 hidden layer neurons, and one output layer neuron. The neural network weights were estimated online according to the adaptive algorithm in (23).

\section{B. Results and Discussion}

The knee/lower limb tracking results for a representative subject with stimulation from the RISE and the NN+RISE controllers are shown in Fig. 1 and are summarized in Table I. In
Table I, the maximum steady-state voltage (SSV) and maximum steady-state error (SSE) are defined as the computed voltage and absolute value of error respectively, that occur after $1.5 \mathrm{~s}$ of the trial. Paired one tailed t-tests (across the subject group) were performed with a level of significance set at $\alpha=0.05$. The results indicate that the developed controller demonstrates the ability of the knee angle to track a desired trajectory with a mean (for eleven tests) RMS error of 2.92 degrees with a mean maximum steady state error of 7.01 degrees. Combining the NN with the RISE feedback structure in [13] yields (statistically significant) reduced mean RMS error for approximately the same input stimulus. The maximum steady state voltages for the RISE and NN+RISE controllers revealed no statistical differences.

To illustrate that the performance of the NN+RISE controller (in comparison to the RISE controller alone) can be more significant for different desired trajectories, both controllers were implemented on three subjects (two male, one female) with the control objective to track a dual periodic (4-6 s) desired trajectory with a higher range of motion. The stimulation results from the RISE and the NN+RISE controllers are shown in Fig. 2 and are summarized in Table II. In Table II, the maximum SSV and SSE were observed after $4 \mathrm{~s}$ of the trial. The results illustrate that the NN+RISE controller yields reduced mean RMS error (across the group) and reduced mean maximum SSE (across the group) for approximately the same input stimulus. Paired one tailed t-tests (across the subject group) were performed with a level of significance set at $\alpha=0.05$. The results show that the difference in mean RMS error and mean maximum SSE were statistically significant. The P value for the mean RMS error 
TABLE I

Summarized EXPerimental Results and P VAlues of ONe TAILEd Paired T-Test For a 1.5 s Period Desired Trajectory.

* INDiCATES STATISTICAL DiFFERENCE

\begin{tabular}{|c|c|c|c|c|c|c|c|c|c|}
\hline \multirow[t]{2}{*}{ Subject } & \multirow[t]{2}{*}{ Leg } & \multicolumn{2}{|c|}{ RMS Error } & \multicolumn{2}{|c|}{ Max SSE } & \multicolumn{2}{|c|}{ Mean Voltage [Volts] } & \multicolumn{2}{|c|}{ Max SSV [Volts] } \\
\hline & & RISE & NN+RISE & RISE & NN+RISE & RISE & NN+RISE & RISE & NN+RISE \\
\hline $\mathrm{A}$ & Left & $3.59^{\circ}$ & $2.92^{\circ}$ & $12.42^{\circ}$ & $7.59^{\circ}$ & 22.91 & 23.98 & 29.5 & 31 \\
\hline A & Right & $2.60^{\circ}$ & $2.63^{\circ}$ & $5.74^{\circ}$ & $6.51^{\circ}$ & 27.70 & 25.40 & 32.95 & 31.5 \\
\hline $\mathrm{B}$ & Left & $2.47^{\circ}$ & $2.23^{\circ}$ & $5.95^{\circ}$ & $4.24^{\circ}$ & 22.41 & 22.81 & 28.1 & 26.95 \\
\hline B & Right & $2.83^{\circ}$ & $2.74^{\circ}$ & $6.28^{\circ}$ & $6.76^{\circ}$ & 25.10 & 23.03 & 29.8 & 30.5 \\
\hline $\mathrm{C}$ & Left & $3.18^{\circ}$ & $2.46^{\circ}$ & 8.1 & $6.17^{\circ}$ & 41.35 & 40.14 & 48.9 & 44.8 \\
\hline $\mathrm{C}$ & Right & $2.97^{\circ}$ & $3.01^{\circ}$ & $6.9^{\circ}$ & $9.63^{\circ}$ & 36.32 & 35.15 & 46.4 & 42.3 \\
\hline $\mathrm{D}$ & Left & $3.23^{\circ}$ & $3.71^{\circ}$ & $6.04^{\circ}$ & $5.86^{\circ}$ & 25.25 & 28.24 & 30 & 34.1 \\
\hline $\mathrm{D}$ & Right & $3.53^{\circ}$ & $2.96^{\circ}$ & $8.8^{\circ}$ & $7.58^{\circ}$ & 13.62 & 14.95 & 24.2 & 23.4 \\
\hline $\mathrm{E}$ & Left & $3.92^{\circ}$ & $3.26^{\circ}$ & $11.15^{\circ}$ & $7.92^{\circ}$ & 30.89 & 31.46 & 45 & 40.5 \\
\hline $\mathrm{F}$ & Left & $3.38^{\circ}$ & $2.83^{\circ}$ & $7.99^{\circ}$ & $6.41^{\circ}$ & 26.15 & 28.13 & 31.8 & 34.1 \\
\hline $\mathrm{G}$ & Left & $3.52^{\circ}$ & $3.32^{\circ}$ & $8.2^{\circ}$ & $8.45^{\circ}$ & 41.59 & 43.44 & 49.8 & 50 \\
\hline Mean & & $3.20^{\circ}$ & $* 2.92^{\circ}$ & $7.96^{\circ}$ & $7.01^{\circ}$ & 28.48 & 28.79 & 36.04 & 35.38 \\
\hline Std. Dev. & & $0.45^{\circ}$ & $0.41^{\circ}$ & $2.18^{\circ}$ & $1.44^{\circ}$ & 8.49 & 8.29 & 9.44 & 8.08 \\
\hline $\mathbf{P}(\mathbf{T}<=\mathbf{t})$ & & \multicolumn{2}{|c|}{0.02} & \multicolumn{2}{|c|}{0.08} & \multicolumn{2}{|r|}{0.28} & \multicolumn{2}{|r|}{0.22} \\
\hline
\end{tabular}
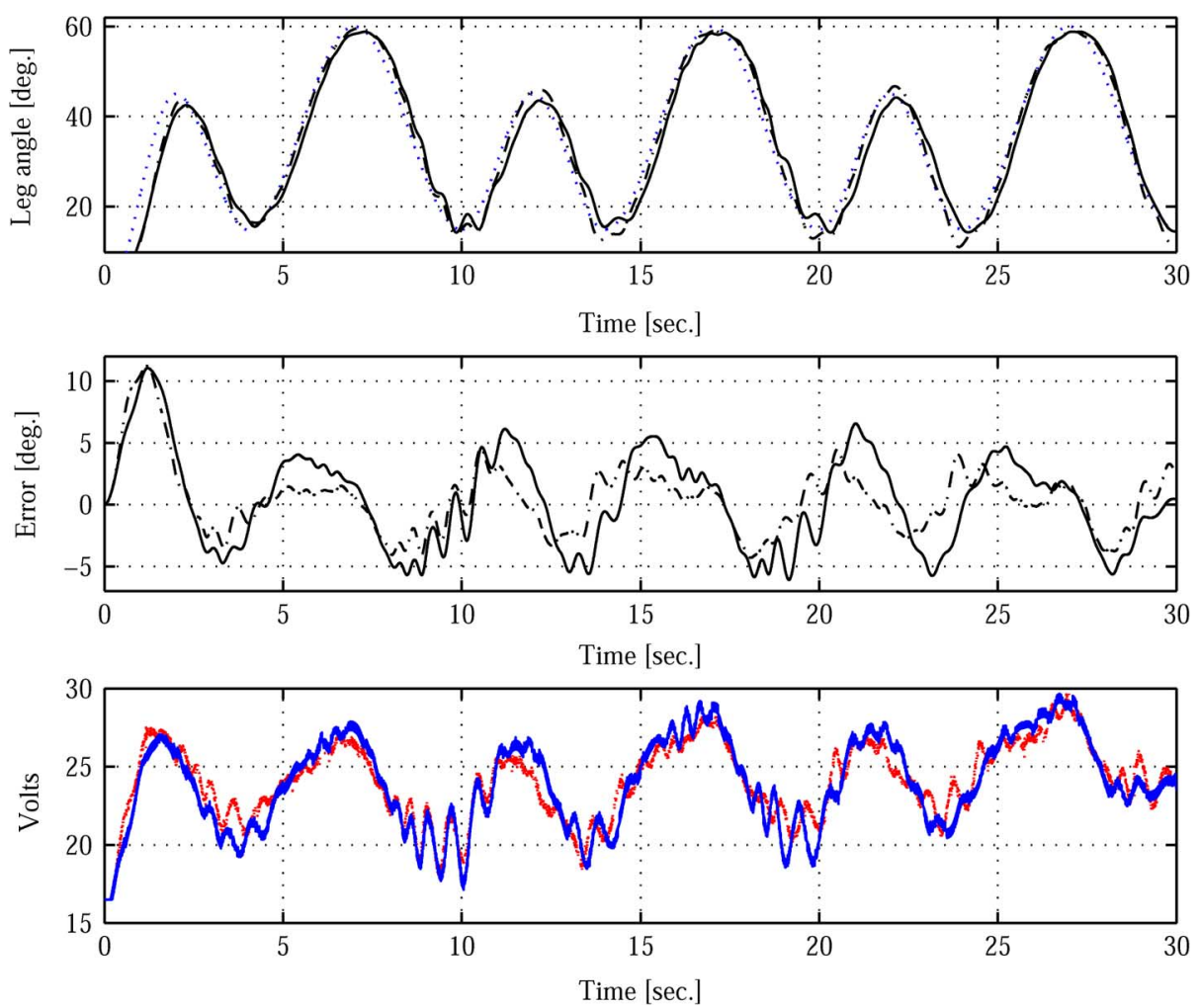

Fig. 2. Top plot shows the actual limb trajectories obtained from the NN+RISE controller (dashed line) and the RISE controller (solid line) versus the dual periodic desired trajectory (dotted line). The middle plot shows the tracking error (desired angle minus actual angle) obtained from NN+RISE (dashed line) and RISE (solid line) controllers. The maximum steady state errors obtained are $4.57^{\circ}$ (at $10.5 \mathrm{~s}$ ) and $6.56^{\circ}$ (at $21 \mathrm{~s}$ ) for NN+RISE and RISE controller, respectively. The bottom plot shows the computed NN+RISE voltage (dotted line) and RISE voltage (solid line). The maximum SSV obtained are 29.68 (at 26.9 s) and 29.67 $\mathrm{V}$ (at $26.7 \mathrm{~s}$ ) for NN+RISE and RISE controller, respectively.

(0.00043) and mean maximum SSE (0.0033) t-test obtained in the case of dual periodic trajectory is smaller when compared to the $\mathrm{P}$ values ( 0.02 and 0.08 , respectively) obtained for the 1.5 $\mathrm{s}$ trajectory. This difference indicates the increased role of the $\mathrm{NN}$ for slower trajectories (where the adaptation gains can be increased).

As in [13], additional experiments were also conducted to examine the performance of the NN+RISE controller in response to step changes and changing loads. Specifically, a desired trajectory of a step input was commanded with a $10 \mathrm{lb}$ load attached to the LEM. An additional $10 \mathrm{lb}$ load was added once the limb stabilized at 15 degrees. The limb was again commanded to perform a step response to raise the limb back up an additional 15 degrees with the total load of $20 \mathrm{lb}$. The results using the NN+RISE controller are shown in Fig. 3. The experimental results for the step response and load addition are given in Table III. The results give some indication of the controller's ability to adapt to changes in load and step inputs and motivate possible future case studies.

Experiments were also performed to test the NN+RISE controller for a sit-to-stand task. These tests were conducted on a non-impaired individual initially seated on a chair. The objec- 
TABLE II

Summarized Experimental Results and P Values of One TAiled Paired T-Test For Dual Periodic (4-6 s) Desired Trajectory. * INDiCATES STATISTICAL DiFFERENCE

\begin{tabular}{|c|c|c|c|c|c|c|c|c|c|}
\hline Subject & Leg & \multicolumn{2}{|c|}{ RMS Error } & \multicolumn{2}{|c|}{ Max SSE } & \multicolumn{2}{|c|}{ Mean Voltage [Volts] } & \multicolumn{2}{|c|}{ Max SSV [Volts] } \\
\hline & & RISE & NN+RISE & RISE & NN+RISE & RISE & NN+RISE & RISE & NN+RISE \\
\hline $\mathrm{A}$ & Left & $2.35^{\circ}$ & $1.85^{\circ}$ & $6.12^{\circ}$ & $4.30^{\circ}$ & 29.08 & 29.19 & 34.10 & 34.09 \\
\hline A & Right & $1.73^{\circ}$ & $1.26^{\circ}$ & $4.49^{\circ}$ & $3.9^{\circ}$ & 30.00 & 29.67 & 35.75 & 34.62 \\
\hline B & Left & $3.52^{\circ}$ & $2.62^{\circ}$ & $6.45^{\circ}$ & $5.64^{\circ}$ & 37.09 & 36.34 & 44.04 & 43.47 \\
\hline B & Right & $3.39^{\circ}$ & $2.89^{\circ}$ & $6.53^{\circ}$ & $6.00^{\circ}$ & 37.88 & 38.57 & 45.30 & 46.19 \\
\hline $\mathrm{C}$ & Right & $3.84^{\circ}$ & $2.82^{\circ}$ & $6.56^{\circ}$ & $4.57^{\circ}$ & 23.99 & 24.09 & 29.67 & 29.68 \\
\hline Mean & & $2.97^{\circ}$ & $* 2.29^{\circ}$ & $6.03^{\circ}$ & $* 4.88^{\circ}$ & 31.61 & 31.57 & 37.77 & 37.61 \\
\hline Std. Dev. & & $\mathbf{0 . 8 9}^{\circ}$ & $0.71^{\circ}$ & $0.88^{\circ}$ & $0.90^{\circ}$ & 5.84 & 5.85 & 6.69 & 6.93 \\
\hline $\mathbf{P}(\mathbf{T}<=\mathbf{t})$ & & \multicolumn{2}{|c|}{0.00043} & \multicolumn{2}{|c|}{0.0033} & \multicolumn{2}{|r|}{0.43} & \multicolumn{2}{|r|}{0.29} \\
\hline
\end{tabular}
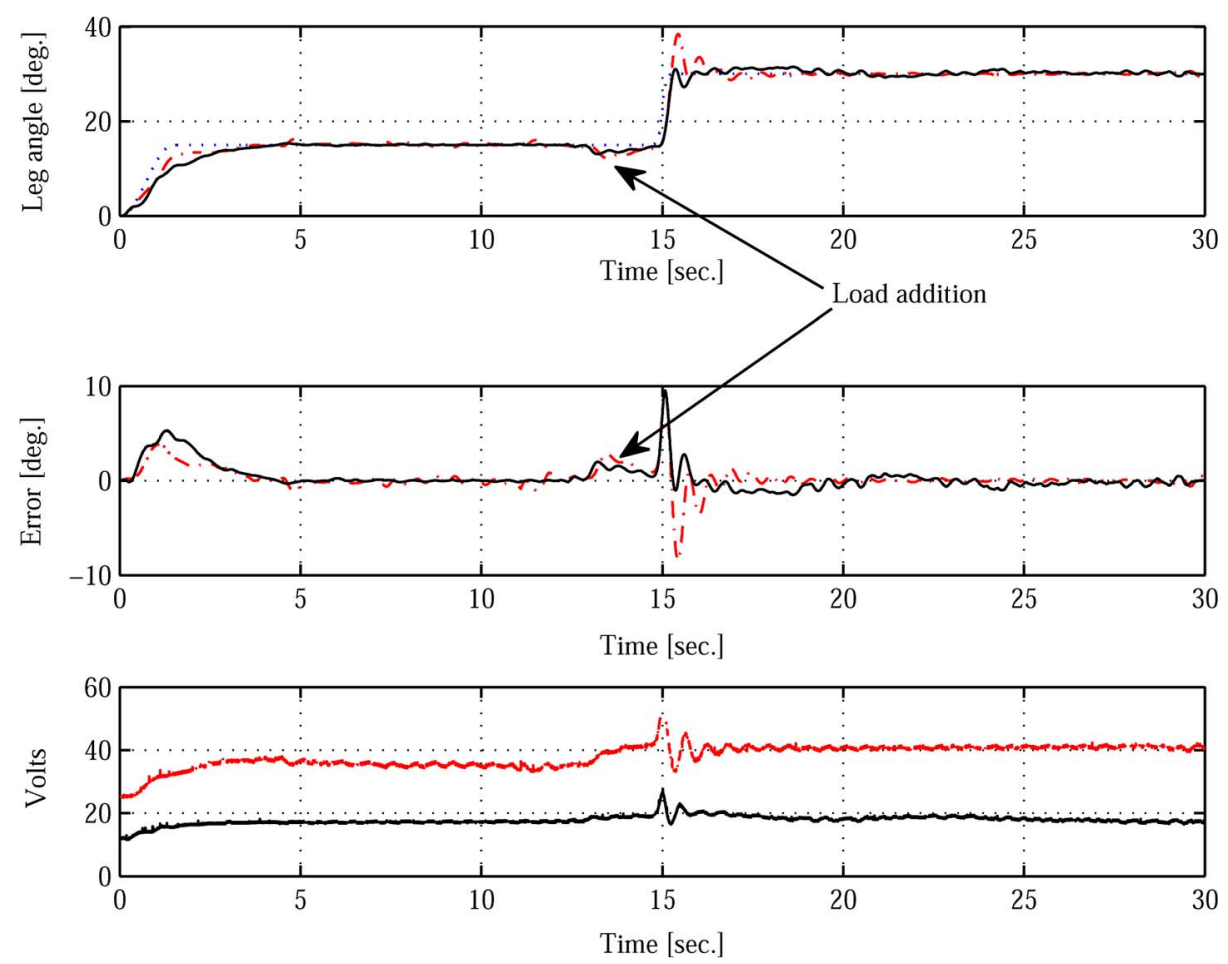

Fig. 3. Experimental plots (subject A-dashed-dotted line; subject B-solid line) for step change and load addition obtained from the NN+RISE controller. The top plot shows actual limb trajectory versus the desired step trajectory (dotted line). The load was added once the limb stabilizes (between 13-15 $\mathrm{s}$ interval). After the load addition the limb was tested for the step input. The middle plot shows the limb tracking error obtained during the experiment. The bottom plot shows computed voltage for the experiment.

TABLE III

EXPERIMENTAL RESULTS FOR STEP RESPONSE AND CHANGING LOADS

\begin{tabular}{|c|c|lc|c|l|l|}
\hline Subject & Leg & $\begin{array}{l}\text { Max. SSE (after } \\
\text { step input) }\end{array}$ & Max. Transient Error & $\begin{array}{l}\text { Max. Error (during } \\
\text { disturbance) }\end{array}$ & $\begin{array}{l}\text { Max. SSV (after } \\
\text { step input) [Volts] }\end{array}$ \\
\hline A & Left & $0.7^{\circ}$ & $9.5^{\circ}$ & $2.8^{\circ}$ & 42.2 \\
\hline B & Right & $0.6^{\circ}$ & $9.52^{\circ}$ & $2.0^{\circ}$ & 19.2 \\
\hline
\end{tabular}

tive was to control the angular knee trajectory that resulted in the volunteer rising from a seated position, with a final desired angle of $90^{\circ}$ (standing position) and the initial knee angle of $0^{\circ}$ (sitting position). The error, voltage, and desired versus actual knee angle plots are shown in Fig. 4. The final SSE is within $-0.5^{\circ}$, the maximum transient error was observed as $8.23^{\circ}$ (at $1.64 \mathrm{~s}$ ), and the maximum voltage was obtained as $35.1 \mathrm{~V}$ (at $1.59 \mathrm{~s}$ ). The RMS error and the mean voltage were obtained as $2.92^{\circ}$ and $26.88 \mathrm{~V}$, respectively.

The NN+RISE structure is motivated by the desire to blend a NN-based feedforward method with a continuous feedback
RISE structure to obtain asymptotic limb tracking despite an uncertain nonlinear muscle response. The ability of the neural networks to learn uncertain and unknown muscle dynamics is complemented by the ability of RISE to compensate for additive system disturbances (hyperactive somatosensory reflexes that may be present in impaired individuals) and NN approximation error. Although the NN+RISE controller was successfully implemented and compared to RISE controller in the present work, the performance of the controller may be further improved in efforts to reduce the effects of muscle fatigue in future studies. Fatigue can be reduced for short 

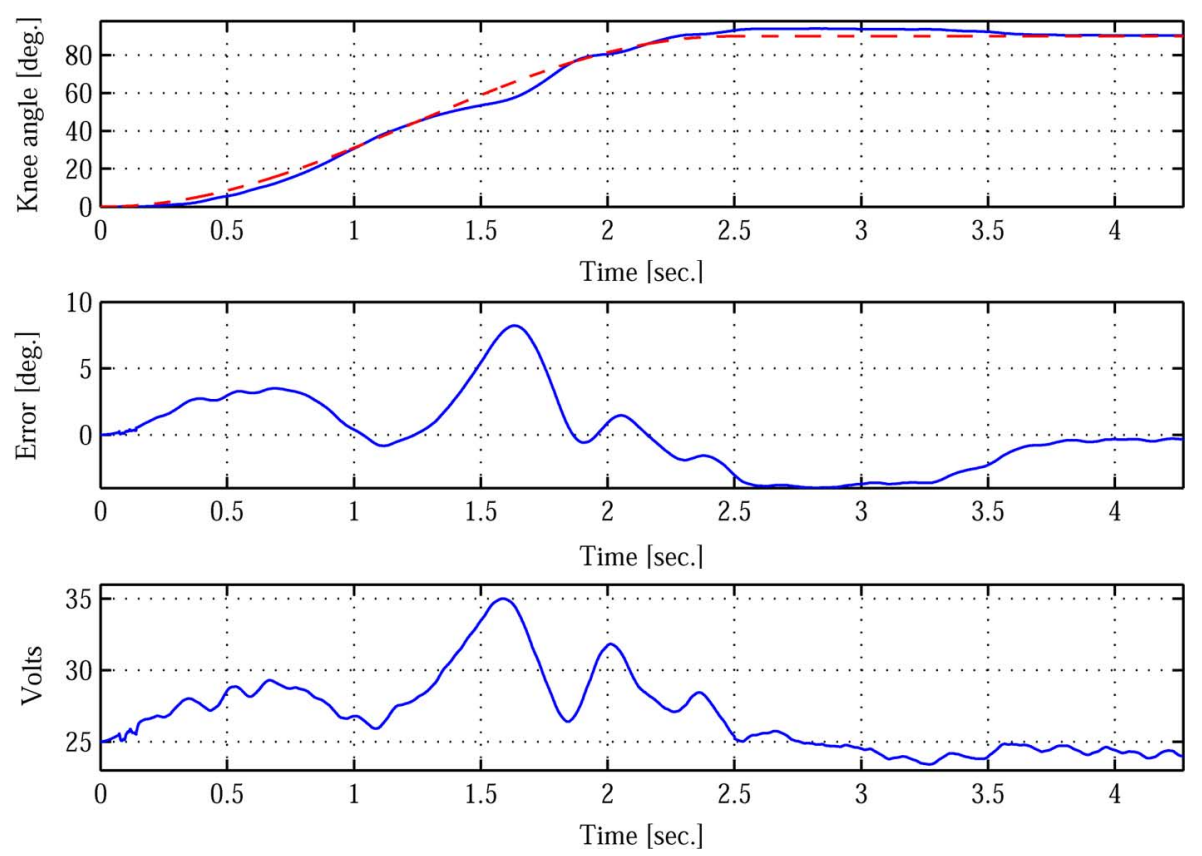

Fig. 4. Top plot shows the actual leg angle trajectory (solid line) versus desired trajectory (dashed line) obtained during the standing experiment. The middle plot shows the error obtained during the experiment. The bottom plot shows the voltage produced during the experiment.

durations by selecting optimal stimulation parameters, but FES/NMES may require a controller that adapts with fatigue to yield performance gains for longer time durations. Therefore, future development includes the use of a fatigue model in the muscle dynamics as a means to provide desired results for longer durations.

\section{Limitations}

The results illustrate the added value of including a NN feedforward component in comparison to only using the RISE feedback structure in [13]. However, several limitations exist in the experimental study. The contribution from the NN component in the case of $1.5 \mathrm{~s}$ periodic desired trajectory was observed to increase but the RISE contribution did not decline proportionally. On the other hand, respective contributions from the RISE and NN component in the dual periodic desired trajectory case were relatively stationary, and the NN component's contribution was found to be relatively larger in this case. As a representative example, Fig. 5 shows the results obtained from a same subject for two cases: $1.5 \mathrm{~s}$ desired trajectory and dual periodic desired trajectory, where it depicts the individual contributions of NN and RISE components in the applied voltages. The ratios of NN and RISE contributions in the Fig. 5 for $1.5 \mathrm{~s}$ period desired trajectory and dual periodic desired trajectory were obtained as 0.088 and 0.165 , respectively, which were calculated as RMS NN voltage over mean RISE voltage. A possible reason for this observation is that the $1.5 \mathrm{~s}$ period desired trajectory has a large desired acceleration $\ddot{q}_{d}(t)$, which is an input to the $\mathrm{NN}$ that can lead to large voltage swings during the transient stage. To reduce large voltage variants during the transient due to $\ddot{q}_{d}(t)$, the update law gains are reduced in comparison to gains that could be employed during less aggressive trajectories. Also, the experimental results with slower trajectories (dual periodic-4-6 s period) illustrate that the NN component can play a larger role depending on the trajectory. Specifically, the dual periodic trajectory results indicate that the RMS error obtained with the NN+RISE controller is lower than the RMS error obtained with the RISE controller with a lower $P$ value $(0.00043)$ compared to the $\mathrm{P}$ value $(0.02)$ obtained with the $1.5 \mathrm{~s}$ period trajectory.

Since a trajectory for a specific functional task was not provided, the desired trajectory used in the first set of experiments was simply selected as a continuous sinusoid with a constant 1.5 s period. The desired trajectory was arbitrarily selected, but the period of the sinusoid is inspired by a typical walking gait trajectory. As the work transitions to applications where a specific functional trajectory is generated, the control results should directly translate. Furthermore, some clinical goals may be better expressed as a desired force profile rather than a desired limb trajectory. The results from this work could be directly applied to these cases by altering the control objective and open-loop error system, but the form of the control method (i.e., NN+RISE) would remain intact.

For all experiments, the subjects were not aware of the order of the control implementation, and the RISE controller was implemented first so that proper gains could be determined. The $\mathrm{NN}+\mathrm{RISE}$ controller was implemented by simply adding the NN component to the tuned RISE controller. This approach allows a direct comparison that highlights the contribution of the $\mathrm{NN}$ for the same set of control gains. However, the subjects could have been more comfortable or experienced more fatigue when the second set of experiments were performed. Ideally the controllers would have been implemented in a random manner.

The Lyapunov-based analysis provides conservative sufficient gain conditions. The control gains for the experiments were obtained by choosing gains and then adjusting them based on the transient and steady-state performance. If the response exhibited a prolonged transient response (compared with the 

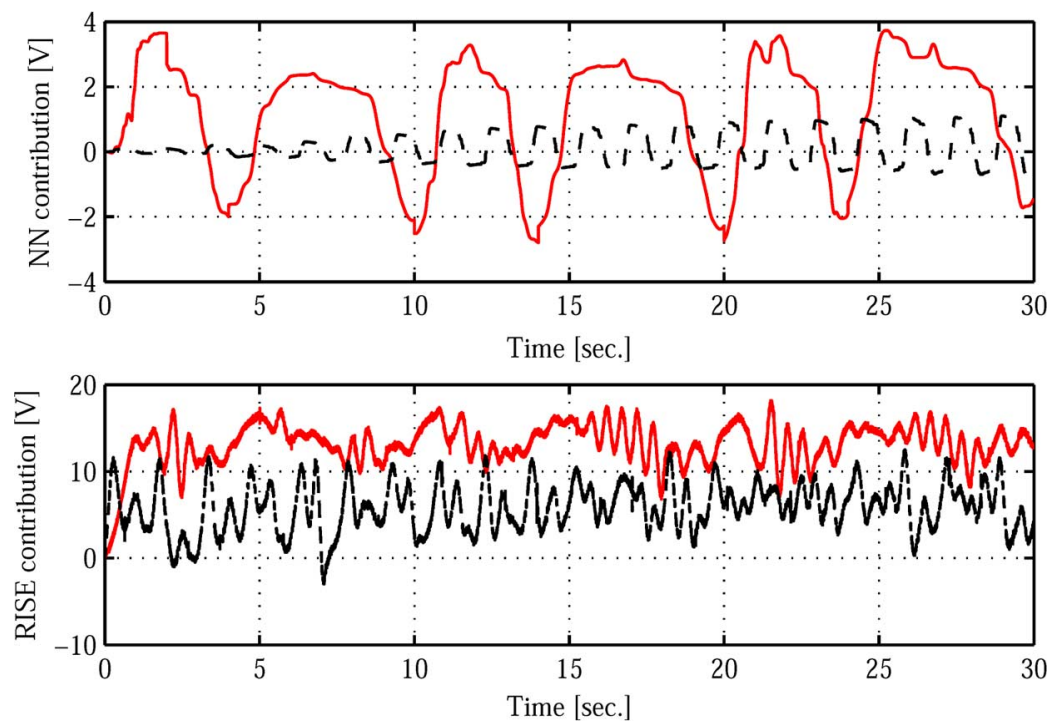

Fig. 5. As a representative example, the figure shows respective contributions from NN and RISE components in the voltages applied to a subject for two cases: $1.5 \mathrm{~s}$ desired trajectory (dashed line) and dual periodic desired trajectory (solid line). The top plot shows the NN contributions while the bottom plot shows the RISE contributions in the respective applied voltages. The NN contribution in the case of 1.5 periodic trajectory increases but the RISE component does not decrease proportionally. However, the respective contributions from the RISE and NN component in the dual periodic desired trajectory case are relatively stationary and also, the NN component's contribution is relatively greater in this case.

TABLE IV

Table Shows the RMS ERrors During Extension and FleXion Phase of the Leg Movement Across DifFerent SubJeCts, TRAJeCtories (1.5 s AND Dual Periodic), AND CONTROllers (RISE/NN+RISE). THE RESUlts Show That the MEAN RMS ERROR IS More During the Extension Phase Than During the FleXion PHase

\begin{tabular}{|c|c|c|c|c|c|c|}
\hline Subject & Leg & Trajectory & \multicolumn{2}{|c|}{ RMS Error (RISE) } & \multicolumn{2}{|c|}{ RMS Error (NN+RISE) } \\
\hline & & & Extension & Flexion & Extension & Flexion \\
\hline $\mathbf{A}$ & Left & Dual period & $4.35^{\circ}$ & $2.41^{\circ}$ & $3.30^{\circ}$ & $1.68^{\circ}$ \\
\hline $\mathbf{A}$ & Right & Dual period & $3.98^{\circ}$ & $2.68^{\circ}$ & $3.39^{\circ}$ & $2.28^{\circ}$ \\
\hline B & Left & Dual period & $2.74^{\circ}$ & $1.86^{\circ}$ & $1.77^{\circ}$ & $1.92^{\circ}$ \\
\hline B & Right & Dual period & $1.78^{\circ}$ & $1.69^{\circ}$ & $1.35^{\circ}$ & $1.17^{\circ}$ \\
\hline $\mathrm{C}$ & Right & Dual period & $4.22^{\circ}$ & $3.43^{\circ}$ & $3.27^{\circ}$ & $2.28^{\circ}$ \\
\hline D & Left & 1.5 second & $2.87^{\circ}$ & $2.00^{\circ}$ & $2.54^{\circ}$ & $1.88^{\circ}$ \\
\hline D & Right & 1.5 second & $3.21^{\circ}$ & $2.38^{\circ}$ & $3.07^{\circ}$ & $2.38^{\circ}$ \\
\hline $\mathbf{E}$ & Left & 1.5 second & $3.87^{\circ}$ & $3.30^{\circ}$ & $3.30^{\circ}$ & $2.49^{\circ}$ \\
\hline $\mathbf{E}$ & Right & 1.5 second & $2.56^{\circ}$ & $2.65^{\circ}$ & $2.34^{\circ}$ & $2.88^{\circ}$ \\
\hline $\mathbf{F}$ & Left & 1.5 second & $3.81^{\circ}$ & $2.51^{\circ}$ & $4.00^{\circ}$ & $3.40^{\circ}$ \\
\hline $\mathbf{F}$ & Right & 1.5 second & $3.59^{\circ}$ & 3.47 & $2.96^{\circ}$ & $2.96^{\circ}$ \\
\hline $\mathbf{G}$ & Left & 1.5 second & $3.93^{\circ}$ & $2.18^{\circ}$ & $2.86^{\circ}$ & $1.97^{\circ}$ \\
\hline G & Right & 1.5 second & $2.98^{\circ}$ & $2.95^{\circ}$ & $2.82^{\circ}$ & $3.19^{\circ}$ \\
\hline H & Left & 1.5 second & $4.18^{\circ}$ & $2.70^{\circ}$ & $3.92^{\circ}$ & $2.58^{\circ}$ \\
\hline I & Left & 1.5 second & $3.97^{\circ}$ & $2.66^{\circ}$ & $3.11^{\circ}$ & $2.51^{\circ}$ \\
\hline $\mathbf{J}$ & Right & 1.5 second & $3.79^{\circ}$ & $4.05^{\circ}$ & $3.38^{\circ}$ & $3.13^{\circ}$ \\
\hline Mean & & & $3.49^{\circ}$ & $2.68^{\circ}$ & $2.96^{\circ}$ & $2.42^{\circ}$ \\
\hline $\mathbf{P}(\mathbf{T}<=\mathbf{t})$ & & & 0.000 & & & \\
\hline
\end{tabular}

response obtained with other gains), the proportional gains were adjusted. If the response exhibited overshoot, derivative gains were adjusted. The control gains for the experiments were tuned based on this trial and error basis. In contrast to this trial and error approach, the control gains could have been adjusted using more methodical approaches as described in various survey papers on the topic [38], [39].

An analysis across different subjects and trajectories $(1.5 \mathrm{~s}$ and dual periodic) indicate that the mean RMS error is more during leg extension and flexion. A t-test analysis shows that the results are statistically significant with $p$ values of 0.00013 and 0.0014 obtained from the RISE and NN+RISE controllers, respectively. The mean RMS errors during the extension phase for the RISE and NN+RISE controllers were $3.49^{\circ}$ and $2.68^{\circ}$, re- spectively, while the mean RMS errors during the flexion phase was $2.96^{\circ}$ and $2.42^{\circ}$, respectively. Summarized RMS errors for both phases are shown in Table IV. An increased error during extension phase can be attributed to higher control effort required during extension. The performance during the extension phase can also be aggravated by increased time delay and muscle fatigue due to the requirement for higher muscle force compared to the flexion phase. This analysis indicates a possible need for separate control strategies during extension and flexion phase of the leg movement. Particularly, future efforts will investigate a hybrid control approach for each phase of motion.

Currently the experiments were performed on non-impaired persons. In future studies with impaired individuals, our untested hypothesis is that the added value of the $\mathrm{NN}$ feed- 
forward component will be even more pronounced (and that the controller will remain stable) as disturbances due to more rapid fatigue and more sensitive somatosensory reflexes may be present in impaired individuals. To delay the onset of fatigue, different researchers have proposed different stimulation strategies [40]-[42] such as choosing different stimulation patterns and parameters. The NMES controller in this study was implemented using constant pulse width amplitude modulation of the voltage. However, the controller can be implemented using other modulation schemes such as pulse width and frequency modulation without any implications on the stability analysis, but the effects of using frequency modulation or varying pulse trains (e.g., a pulse train containing doublets) remain to be investigated clinically.

\section{CONCLUSION}

A Lyapunov-based stability analysis indicates that the developed closed-loop nonlinear NMES control method yields asymptotic tracking for a unknown nonlinear muscle activation and limb dynamics, even in the presence of uncertain additive disturbances. Experiments using external electrodes on non-impaired volunteers demonstrated the ability of the $\mathrm{NN}+\mathrm{RISE}$ controller to enable the knee and lower leg to track a desired trajectory composed of sinusoids, step changes, and changes in the load. Statistical analysis of the experimental results indicates that the NN+RISE algorithm yields reduced RMS tracking error when compared to the RISE controller for statistically insignificant differences in voltage input. Future efforts will explore non-quadratic Lyapunov functions and methods based on convex optimization in [43] to improve the current stability analysis.

\section{APPENDIX A}

\section{STABILITY ANALYSIS}

Proof for Theorem 1: Let $\mathcal{D} \subset \mathbb{R}^{5}$ be a domain containing $y(t)=0$, where $y(t) \in \mathbb{R}^{5}$ is defined as

$$
y(t) \triangleq\left[\begin{array}{lll}
z^{T} & \sqrt{P(t)} & \sqrt{Q(t)}
\end{array}\right]^{T}
$$

where the auxiliary function $Q(t) \in \mathbb{R}$ is defined as

$$
Q(t) \triangleq \frac{\alpha_{2}}{2} \operatorname{tr}\left(\tilde{W}^{T} \Gamma_{1}^{-1} \tilde{W}\right)+\frac{\alpha_{2}}{2} \operatorname{tr}\left(\tilde{U}^{T} \Gamma_{2}^{-1} \tilde{U}\right)
$$

and $P(t) \in \mathbb{R}$ is the generalized solution to the differential equation

$$
\dot{P}(t)=-L(t), \quad P(0)=\beta_{1}\left|e_{2}(0)\right|-e_{2}(0) N(0) .
$$

Since $\Gamma_{1}$ and $\Gamma_{2}$ in (41) are constant, symmetric, and positive definite matrices, and $\alpha_{2}>0$, it is straightforward that $Q(t) \geq$ 0 . The auxiliary function $L(t) \in \mathbb{R}$ in (42) is defined as

$$
L(t) \triangleq r\left(N_{B_{1}}+N_{d}-\beta_{1} \operatorname{sgn}\left(e_{2}\right)\right)+\dot{e}_{2} N_{B_{2}}-\beta_{2} e_{2}^{2}
$$

where $\beta_{1}, \beta_{2} \in \mathbb{R}$ introduced in (22) and (43) respectively, are positive constants chosen according to the following sufficient conditions:

$$
\beta_{1}>\zeta_{1}+\zeta_{2}+\frac{1}{\alpha_{2}} \zeta_{3}+\frac{1}{\alpha_{2}} \zeta_{4}, \quad \beta_{2}>\zeta_{5}
$$

where $\zeta_{i} \in R,(i=1,2, \ldots, 5)$ are known positive constants introduced in (38). Provided the sufficient conditions in (44) are satisfied, then $P(t) \geq 0$.

Let $V_{L}(y, t): \mathcal{D} \times[0, \infty) \rightarrow \mathbb{R}$ denote a Lipschitz continuous regular positive definite functional defined as

$$
V_{L}(y, t) \triangleq e_{1}^{2}+\frac{1}{2} e_{2}^{2}+\frac{1}{2} J_{\Omega} r^{2}+P+Q
$$

which satisfies the inequalities

$$
U_{1}(y) \leq V_{L}(y, t) \leq U_{2}(y)
$$

provided the sufficient conditions in (44) are satisfied, where $U_{1}(y), U_{2}(y) \in \mathbb{R}$ are continuous, positive definite functions defined as

$$
U_{1}(y)=\lambda_{1}\|y\|^{2}, \quad U_{2}(y)=\lambda_{2}\|y\|^{2}
$$

where $\lambda_{1}, \lambda_{2} \in \mathbb{R}$ are known positive functions or constants. After taking the time derivative of $(45), \dot{V}_{L}(y, t)$ can be expressed as

$$
\dot{V}_{L}(y, t) \triangleq 2 e_{1} \dot{e}_{1}+e_{2} \dot{e}_{2}+J_{\Omega} r \dot{r}+\frac{1}{2} \dot{J}_{\Omega} r^{2}+\dot{P}+\dot{Q}
$$

From (8), (29), (42), (43), and after taking the time derivative of (41), some of the differential equations describing the closedloop system for which the stability analysis is being performed have discontinuous right-hand sides as

$$
\begin{aligned}
\dot{e}_{1} & =e_{2}-\alpha_{1} e_{1} \\
\dot{e}_{2} & =r-\alpha_{2} e_{2} \\
J_{\Omega} \dot{r} & =-\frac{1}{2} \dot{J}_{\Omega} r+\tilde{N}+N-e_{2}-\left(k_{s}+1\right) r-\beta \operatorname{sgn}\left(e_{2}\right) \\
\dot{P}(t) & =-r\left(N_{B_{1}}+N_{d}-\beta_{1} \operatorname{sgn}\left(e_{2}\right)\right)-\dot{e}_{2} N_{B_{2}}+\beta_{2} e_{2}^{2} \\
\dot{Q}(t) & =\operatorname{tr}\left(\alpha_{2} \tilde{W}^{T} \Gamma_{1}^{-1} \dot{\tilde{W}}\right)+\operatorname{tr}\left(\alpha_{2} \tilde{U}^{T} \Gamma_{2}^{-1} \dot{\tilde{U}}\right)
\end{aligned}
$$

Let $f(y, t) \in \mathbb{R}^{5}$ denote the right-hand side of (47). Since the subsequent analysis requires that a solution exists for $\dot{y}=$ $f(y, t)$, it is important to show the existence of the solution to (47). As described in [44], the existence of Filippov's generalized solution can be established for (47). First, note that $f(y, t)$ is continuous except in the set $\left\{(y, t) \mid e_{2}=0\right\}$. From [44], [45], an absolute continuous Filippov solution $y(t)$ exists almost everywhere (a.e.) so that

$$
\dot{y} \in K[f](y, t) \quad \text { a.e. }
$$


Except the points on the discontinuous surface $\left\{(y, t) \mid e_{2}=\right.$ $0\}$, the Filippov set-valued map includes unique solution. Under Filippov's framework, a generalized Lyapunov stability theory can be used (see [45] and [46] for further details) to establish strong stability of the closed-loop system. The generalized time derivative of (45) exists a.e., and $\dot{V}_{L}(y, t) \in^{\text {a.e. }} \dot{\tilde{V}}_{L}(y, t)$, where

$$
\begin{aligned}
\dot{\tilde{V}}_{L}(y, t) & =\bigcap_{\xi \in \partial V_{L}(y, t)} \xi^{T} K\left[\begin{array}{llllll}
\dot{e}_{1} & \dot{e}_{2} & \dot{r} & \frac{1}{2} P^{-\frac{1}{2}} \dot{P} & \frac{1}{2} Q^{-\frac{1}{2}} \dot{Q} & 1
\end{array}\right]^{T} \\
= & \nabla V_{L}^{T} K\left[\begin{array}{llllll}
\dot{e}_{1} & \dot{e}_{2} & \dot{r} & \frac{1}{2} P^{-\frac{1}{2}} \dot{P} & \frac{1}{2} Q^{-\frac{1}{2}} \dot{Q} & 1
\end{array}\right]^{T} \\
& \subset\left[\begin{array}{llllll}
2 e_{1} & e_{2} & r J_{\Omega} & 2 P^{\frac{1}{2}} & 2 Q^{\frac{1}{2}} & \frac{1}{2} \dot{J}_{\Omega} r^{2}
\end{array}\right] \\
& \times K\left[\begin{array}{llllll}
\dot{e}_{1} & \dot{e}_{2} & \dot{r} & \frac{1}{2} P^{-\frac{1}{2}} \dot{P} & \frac{1}{2} Q^{-\frac{1}{2}} \dot{Q} & 1
\end{array}\right]^{T} .
\end{aligned}
$$

After utilizing (8), (29), (42), (43)

$$
\begin{aligned}
\dot{\tilde{V}}_{L}(y, t) \subset & 2 e_{1} e_{2}-2 \alpha_{1} e_{1}^{2}+e_{2} r-\alpha_{2} e_{2}^{2}+\frac{1}{2} \dot{J}_{\Omega} r^{2} \\
& +r \tilde{N}+r N-r e_{2}-\left(k_{s}+1\right) r^{2}-\beta r K\left[\operatorname{sgn}\left(e_{2}\right)\right] \\
& -\frac{1}{2} \dot{J}_{\Omega} r^{2}-r N_{B_{1}}-r N_{d}+\beta r K\left[\operatorname{sgn}\left(e_{2}\right)\right] \\
& -\dot{e}_{2} N_{B_{2}}+\beta_{2} e_{2}^{2}+\operatorname{tr}\left(\alpha_{2} \tilde{W}^{T} \Gamma_{1}^{-1} \dot{\tilde{W}}\right) \\
& +\operatorname{tr}\left(\alpha_{2} \tilde{U}^{T} \Gamma_{2}^{-1} \dot{\tilde{U}}\right)
\end{aligned}
$$

where [47]

$$
K\left[\operatorname{sgn}\left(e_{2}\right)\right]=\operatorname{SGN}\left(e_{2}\right)
$$

such that

$$
\operatorname{SGN}\left(e_{2}\right)= \begin{cases}1, & e_{2}>0 \\ {[-1,1],} & e_{2}=0 \\ -1, & e_{2}<0\end{cases}
$$

Using (23), (31), (33), and (35), canceling common terms, and based on the fact that

$$
2 e_{1} e_{2} \leq e_{1}^{2}+e_{2}^{2}
$$

(49) can be written as

$$
\begin{aligned}
\dot{\tilde{V}}_{L}(y, t) \subset- & \left(2 \alpha_{1}-1\right) e_{1}^{2} \\
& \quad\left(\alpha_{2}-\beta_{2}-1\right) e_{2}^{2}-r^{2}+r \tilde{N}-k_{s} r^{2} .
\end{aligned}
$$

As shown in (49) and (50), the unique integral signum term in the RISE controller is used to compensate for the disturbance terms included in $N_{d}\left(q_{d}, \dot{q}_{d}, \ddot{q}_{d}, \dddot{q}_{d}, t\right)$ and $N_{B_{1}}\left(\hat{W}, \hat{U}, x_{d}, \dot{x}_{d}, t\right)$, provided the control gain $\beta_{1}$ and $\beta_{2}$ are selected according to (44). Further the term $N_{B_{2}}\left(\hat{W}, \hat{U}, x_{d}, \dot{x}_{d}, t\right)$ is partially rejected by the unique integral signum term and partially canceled by adaptive update law. Using (36), the term $r(t) \tilde{N}\left(e_{1}, e_{2}, r, t\right)$, can be upper bounded by following inequality:

$$
|r \tilde{N}| \leq \rho(\|z\|)|| z|||r|
$$

to obtain

$$
\begin{aligned}
\dot{\tilde{V}}_{L}(y, t) \subset-\min \left\{2 \alpha_{1}-1,\right. & \left.\alpha_{2}-\beta_{2}-1,1\right\}\|z\|^{2} \\
& +\left[\rho(\|z\|)\|z\||r|-k_{s} r^{2}\right] .
\end{aligned}
$$

Completing the squares for the bracketed terms in (51) yields

$$
\begin{aligned}
\dot{\tilde{V}}_{L}(y, t) \subset-\min \left\{2 \alpha_{1}-1, \alpha_{2}-\beta_{2}-1,1\right\}\|z\|^{2} & \\
+ & \frac{\rho^{2}(\|z\|)\|z\|^{2}}{4 k_{s}} .
\end{aligned}
$$

The following expression can be obtained from (52):

$$
\dot{\tilde{V}}_{L}(y, t) \subset-U(y)
$$

where $U(y)=c\|z\|^{2}$, for some positive constant $c \in \mathbb{R}$, is a continuous positive semi-definite function that is defined on the following domain:

$$
\mathcal{D} \triangleq\left\{y \in \mathbb{R}^{5} \mid\|y\| \leq \rho^{-1}\left(2 \sqrt{\lambda_{3} k_{s}}\right)\right\}
$$

where $\lambda_{3} \triangleq \min \left\{2 \alpha_{1}-1, \alpha_{2}-\beta_{2}-1,1\right\}$. Let $\mathcal{S} \subset \mathcal{D}$ denote a set defined as follows:

$$
\mathcal{S} \triangleq\left\{y(t) \subset \mathcal{D} \mid U_{2}(y(t)) \leq \lambda_{1}\left(\rho^{-1}\left(2 \sqrt{\lambda_{3} k_{s}}\right)\right)^{2}\right\}
$$

where $\mathcal{S} \subset \mathcal{D}$ is introduced in Theorem 1 . The region of attraction in (54) can be made arbitrarily large to include any initial conditions by increasing the control gain $k_{s}$ (i.e., a semi-global type of stability result), and hence

$$
c\|z(t)\|^{2} \rightarrow 0 \quad \text { as } t \rightarrow \infty \quad \forall y(0) \in \mathcal{S} .
$$

Based on the definition of $z(t)$ in (37), (55) can be used to show that

$$
\left|e_{1}(t)\right| \rightarrow 0 \quad \text { as } t \rightarrow \infty \quad \forall y(0) \in \mathcal{S} .
$$

\section{REFERENCES}

[1] P. H. Peckham and D. B. Gray, "Functional neuromuscular stimulation," J. Rehab. Res. Dev., vol. 33, pp. 9-11, 1996.

[2] P. H. Peckham and J. S. Knutson, "Functional electrical stimulation for neuromuscular applications," Annu. Rev. Biomed. Eng., vol. 7, pp. 327-360, 2005.

[3] J. J. Abbas and H. J. Chizeck, "Feedback control of coronal plane hip angle in paraplegic subjects using functional neuromuscular stimulation," IEEE Trans. Biomed. Eng., vol. 38, no. 7, pp. 687-698, Jul. 1991.

[4] N. Lan, P. E. Crago, and H. J. Chizeck, "Control of end-point forces of a multijoint limb by functional neuromuscular stimulation," IEEE Trans. Biomed. Eng., vol. 38, no. 10, pp. 953-965, Oct. 1991.

[5] N. Lan, P. E. Crago, and H. J. Chizeck, "Feedback control methods for task regulation by electrical stimulation of muscles," IEEE Trans. Biomed. Eng., vol. 38, no. 12, pp. 1213-1223, Dec. 1991.

[6] T. Schauer, N. O. Negard, F. Previdi, K. J. Hunt, M. H. Fraser, E. Ferchland, and J. Raisch, "Online identification and nonlinear control of the electrically stimulated quadriceps muscle," Control Eng. Pract., vol. 13, pp. 1207-1219, 2005. 
[7] A. H. Vette, K. Masani, and M. R. Popovic, "Implementation of a physiologically identified PD feedback controller for regulating the active ankle torque during quiet stance," IEEE Trans. Neural Syst. Rehab. Eng., vol. 15, no. 2, pp. 235-243, Jun. 2007.

[8] K. Hunt and M. Munih, "Feedback control of unsupported standing in paraplegia-part 1: Optimal control approach," IEEE Trans. Rehab. Eng., vol. 5, no. 4, pp. 331-340, Dec. 1997.

[9] F. Previdi, M. Ferrarin, S. Savaresi, and S. Bittanti, "Gain scheduling control of functional electrical stimulation for assisted standing up and sitting down in paraplegia: A simulation study," Int. J. Adapt. Control Signal Process., vol. 19, pp. 327-338, 2005.

[10] H. Gollee, K. Hunt, and D. Wood, "New results in feedback control of unsupported standing in paraplegia," IEEE Trans. Neural Syst. Rehab. Eng., vol. 12, no. 1, pp. 73-91, Mar. 2004.

[11] S. Jezernik, R. Wassink, and T. Keller, "Sliding mode closed-loop control of FES: Controlling the shank movement," IEEE Trans. Biomed. Eng., vol. 51, no. 2, pp. 263-272, Feb. 2004.

[12] M. Ebrahimpour and A. Erfanian, "Comments on "sliding mode closed-loop control of FES: Controlling the shank movement"," IEEE Trans. Biomed. Eng., vol. 55, no. 12, p. 2842, Dec. 2008.

[13] N. Sharma, K. Stegath, C. M. Gregory, and W. E. Dixon, "Nonlinear neuromuscular electrical stimulation tracking control of a human limb," IEEE Trans. Neural Syst. Rehab. Eng., vol. 17, no. 6, pp. 576-584, Dec. 2009.

[14] N. Lan, H. Feng, and E. Crago, "Neural network generation of muscle stimulation patterns for control of arm movements," IEEE Trans. Rehab. Eng., vol. 2, no. 4, pp. 213-224, Dec. 1994.

[15] J. J. Abbas and H. J. Chizeck, "Neural network control of functional neuromuscular stimulation systems: Computer simulation studies," IEEE Trans. Biomed. Eng., vol. 42, no. 11, pp. 1117-1127, Nov. 1995.

[16] D. Graupe and H. Kordylewski, "Artificial neural network control of FES in paraplegics for patient responsive ambulation," IEEE Trans. Biomed. Eng., vol. 42, no. 7, pp. 699-707, Jul. 1995.

[17] G.-C. Chang, J.-J. Lub, G.-D. Liao, J.-S. Lai, C.-K. Cheng, B.-L. Kuo, and T.-S. Kuo, "A neuro-control system for the knee joint position control with quadriceps stimulation," IEEE Trans. Rehab. Eng., vol. 5, no. 1, pp. 2-11, Mar. 1997.

[18] J. A. Riess and J. J. Abbas, "Adaptive neural network control of cyclic movements using functional neuromuscular stimulation," IEEE Trans. Neural Syst. Rehab. Eng., vol. 8, no. 1, pp. 42-52, Mar. 2000.

[19] H. Kordylewski and D. Graupe, "Control of neuromuscular stimulation for ambulation by complete paraplegics via artificial neural networks," Neurol. Res., vol. 23, no. 5, pp. 472-481, 2001.

[20] J. P. Giuffrida and P. E. Crago, "Functional restoration of elbow extension after spinal-cord injury using a neural network-based synergistic FES controller," IEEE Trans. Neural Syst. Rehab. Eng., vol. 13, no. 2, pp. 147-152, Jun. 2005.

[21] K. Kurosawa, R. Futami, T. Watanabe, and N. Hoshimiya, "Joint angle control by FES using a feedback error learning controller," IEEE Trans. Neural Syst. Rehab. Eng., vol. 13, no. 3, pp. 359-371, Sep. 2005.

[22] A. Pedrocchi, S. Ferrante, E. De Momi, and G. Ferrigno, "Error mapping controller: A closed loop neuroprosthesis controlled by artificial neural networks," J. Neuroeng. Rehab., vol. 3, no. 1, p. 25, 2006.

[23] S. Kim, M. Fairchild, A. Iarkov, J. Abbas, and R. Jung, "Adaptive control for neuromuscular stimulation-assisted movement therapy in a rodent model," IEEE Trans. Biomed. Eng., vol. 56, no. 2, pp. 452-461, Feb. 2008.

[24] A. Ajoudani and A. Erfanian, "A neuro-sliding-mode control with adaptive modeling of uncertainty for control of movement in paralyzed limbs using functional electrical stimulation," IEEE Trans. Biomed. Eng., vol. 56, no. 7, pp. 1771-1780, Jul. 2009.

[25] J. Lujan and P. Crago, "Automated optimal coordination of multiple-DOF neuromuscular actions in feedforward neuroprostheses," IEEE Trans. Biomed. Eng., vol. 56, no. 1, pp. 179-187, Jan. 2009.

[26] P. M. Patre, W. MacKunis, K. Kaiser, and W. E. Dixon, "Asymptotic tracking for uncertain dynamic systems via a multilayer neural network feedforward and RISE feedback control structure," IEEE Trans. Autom. Control, vol. 53, no. 9, pp. 2180-2185, Sep. 2008.

[27] N. Sharma, C. M. Gregory, M. Johnson, and W. E. Dixon, "Modified neural network-based electrical stimulation for human limb tracking," in Proc. IEEE Int. Symp. Intell. Control, 2008, pp. 1320-1325.

[28] M. Ferrarin and A. Pedotti, "The relationship between electrical stimulus and joint torque: A dynamic model," IEEE Trans. Rehab. Eng., vol. 8, no. 3, pp. 342-352, Sep. 2000.

[29] J. L. Krevolin, M. G. Pandy, and J. C. Pearce, "Moment arm of the patellar tendon in the human knee," J. Biomech., vol. 37, pp. 785-788, 2004.
[30] W. L. Buford, Jr., F. M. Ivey, Jr., J. D. Malone, R. M. Patterson, G. L. Peare, D. K. Nguyen, and A. A. Stewart, "Muscle balance at the knee-Moment arms for the normal knee and the ACL-Minus knee," IEEE Trans. Rehab. Eng., vol. 5, no. 4, pp. 367-379, Mar. 1997.

[31] Y. Giat, J. Mizrahi, and M. Levy, "A musculotendon model of the fatigue profiles of paralyzed quadriceps muscle under FES," IEEE Trans. Biomed. Eng., vol. 40, no. 7, pp. 664-674, Jul. 1993.

[32] R. Nathan and M. Tavi, "The influence of stimulation pulse frequency on the generation of joint moments in the upper limb," IEEE Trans. Biomed. Eng., vol. 37, no. 3, pp. 317-322, Mar. 1990.

[33] T. Watanabe, R. Futami, N. Hoshimiya, and Y. Handa, "An approach to a muscle model with a stimulus frequency-force relationship for FES applications," IEEE Trans. Rehab. Eng., vol. 7, no. 1, pp. 12-17, Jan. 1999.

[34] F. L. Lewis, R. Selmic, and J. Campos, Neuro-Fuzzy Control of Industrial Systems With Actuator Nonlinearities. Philadelphia, PA: Society for Industrial and Applied Mathematics, 2002.

[35] B. Xian, M. de Queiroz, and D. Dawson, "A continuous control mechanism for uncertain nonlinear systems," in Optimal Control, Stabilization and Nonsmooth Analysis, ser. Lecture Notes in Control and Information Sciences. Heidelberg, Germany: Springer, 2004, vol. 301, pp. 251-264.

[36] P. M. Patre, W. MacKunis, C. Makkar, and W. E. Dixon, "Asymptotic tracking for systems with structured and unstructured uncertainties," IEEE Trans. Control Syst. Technol., vol. 16, no. 2, pp. 373-379, Mar. 2008.

[37] Z. Qu and J. X. Xu, "Model-based learning controls and their comparisons using lyapunov direct method," Asian J. Control, vol. 4, no. 1, pp. 99-110, 2002.

[38] N. J. Killingsworth and M. Krstic, "PID tuning using extremum seeking: online, model-free performance optimization," IEEE Control Syst. Mag., vol. 26, no. 1, pp. 70-79, Jan. 2006.

[39] K. Strm, T. Hgglund, C. Hang, and W. Ho, "Automatic tuning and adaptation for PID controllers-A survey," Control Eng. Pract., vol. 1, no. 4, pp. 669-714, 1993.

[40] R. Maladen, R. Perumal, A. Wexler, and S. Binder-Macleod, "Effects of activation pattern on nonisometric human skeletal muscle performance," J. Appl. Physiol., vol. 102, no. 5, pp. 1985-91, 2007.

[41] S. Binder-Macleod, J. Dean, and J. Ding, "Electrical stimulation factors in potentiation of human quadriceps femoris," Muscle Nerve, vol. 25, no. 2, pp. 271-9, 2002.

[42] C. M. Gregory, W. E. Dixon, and C. S. Bickel, "Impact of varying pulse frequency and duration on muscle torque production and fatigue," Muscle Nerve, vol. 35, no. 4, pp. 504-509, 2007.

[43] G. Chesi, A. Garulli, A. Tesi, and A. Vicino, Homogeneous Polynomial Forms for Robustness Analysis of Uncertain Systems. New York: Springer-Verlag, 2009

[44] A. Filippov, "Differential equations with discontinuous right-hand side," Amer. Math. Soc. Transl., vol. 42, no. 2, pp. 199-231, 1964.

[45] J. P. Aubin and H. Frankowska, Set-Valued Analysis. Boston, MA: Birkhuser, 2008.

[46] D. Shevitz and B. Paden, "Lyapunov stability theory of nonsmooth systems," IEEE Trans. Autom. Control, vol. 39, no. 9, pp. 1910-1914, Sep. 1994.

[47] B. Paden and S. Sastry, "A calculus for computing Filippov's differential inclusion with application to the variable structure control of robot manipulators," IEEE Trans. Circuits Syst., vol. 34, no. 1, pp. 73-82, Jan. 1987.

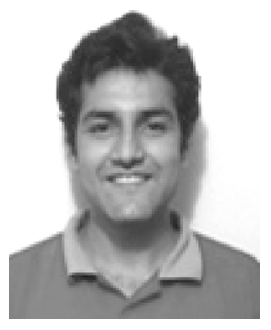

Nitin Sharma (M'10) received the Ph.D. degree from the Department of Mechanical and Aerospace Engineering, University of Florida, Gainesville, in 2010.

Currently, he is a postdoctoral fellow with the Department of Physiology, University of Alberta, Edmonton, $\mathrm{AB}$, Canada. His research interests include intelligent and robust control of functional electrical stimulation (FES), modeling, optimization, and control of FES-elicited walking, and control of uncertain nonlinear systems with input and state delays.

Dr. Sharma was a recipient of 2009 O. Hugo Schuck Award and Best Student Paper Award in Robotics at the 2009 ASME Dynamic Systems and Controls Conference. He was also a finalist for the Best Student Paper Award at the 2008 IEEE Multi-Conference on Systems and Control. 


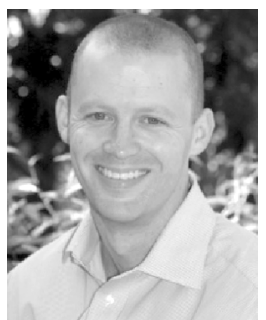

Chris M. Gregory received the Ph.D. degree in exercise physiology from the University of Georgia, Atlanta, in 2002 and completed subsequent postdoctoral training in muscle biology at the University of Florida, Gainesville.

$\mathrm{He}$ is currently an Assistant Professor with the Medical University of South Carolina and a Research Scientist at the Ralph H. Johnson VA Medical Center, Charleston, SC. His research focuses on the study of skeletal muscle form and function. His research has been funded by the Department of Veterans Affairs, the National Institutes of Health as well as the South Carolina Spinal Cord Research Fund. During his career, he has published over 80 manuscripts, conference proceedings, book chapters and abstracts on topics including muscle physiology, biochemistry, exercise and electrical stimulation of skeletal muscle.

Dr. Gregory was a recipient of an Associate Investigator Award and a Career Development Award (level II) from the Department of Veterans Affairs in 2005.

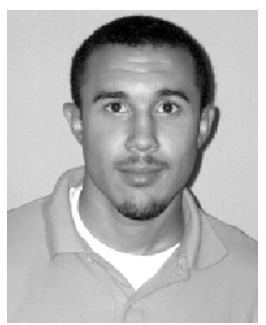

Marcus Johnson (M'10) received the M.S. degree from the Department of Mechanical and Aerospace Engineering, University of Florida, Gainesville, in 2008, where he is currently pursuing the Ph.D. degree.

His main research interest is the development of Lyapunov-based proofs for optimality and stability of nonlinear systems.

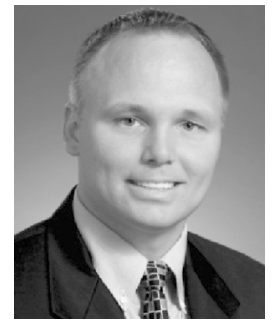

Warren E. Dixon (SM'05) received the Ph.D. degree from the Department of Electrical and Computer Engineering, Clemson University, Clemson, SC, in 2000.

He was a Eugene P. Wigner Fellow with Oak Ridge National Laboratory (ORNL) until joining the University of Florida Mechanical and Aerospace Engineering Department in 2004. His research interests include the development and application of Lyapunov-based control techniques for uncertain nonlinear systems.

Dr. Dixon's work has been recognized by the 2009 O. Hugo Schuck Award, 2006 IEEE Robotics and Automation Society (RAS) Early Academic Career Award, an NSF CAREER Award (2006-2011), 2004 DOE Outstanding Mentor Award, and the 2001 ORNL Early Career Award for Engineering Achievement. $\mathrm{He}$ is an Associate Editor for the ASME Journal of Dynamic Systems, Measurement and Control, Automatica, the IEEE TRANSACTIONS ON SYSTEMS MAN AND CYBERNETICS: PART B CYBERNETICS, the International Journal of Robust and Nonlinear Control, and the Journal of Robotics. 